\title{
Braindrain und Bologna-Drain - Räumliche Implikationen der Bologna-Reform und Auswirkungen auf ländliche Räume. Eine Analyse am Beispiel der Justus-Liebig-Universität Gießen
}

\author{
Philipp Gareis ${ }^{1}$ Christian Diller ${ }^{1}$ Henning Huchthausen ${ }^{1}$ \\ Eingegangen: 7. April 2017 / Angenommen: 23. März 2018 / Online publiziert: 17. April 2018 \\ (c) Springer-Verlag GmbH Deutschland, ein Teil von Springer Nature 2018
}

\section{Zusammenfassung}

Der Beitrag geht den Gründen und räumlichen Verteilungsmustern der Hochschulstandortwahl von Studierenden und den damit verbundenen Auswirkungen auf die Regionalentwicklung, insbesondere in den ländlichen Räumen, nach. Zunächst wird dazu der internationale Stand der Forschung zum Standortwahlverhalten von Studierenden zu verschiedenen Zeitpunkten ihrer Ausbildung dargestellt. Eine eigene regionalstatistische Auswertung kommt für Deutschland zu dem Befund, dass es den Hochschulen in ländlichen Regionen zwar nicht gelingt, einen ähnlich hohen Studierendenbesatz zu erreichen wie in verdichteteren Raumtypen, jedoch die Regionen mit einer Hochschule deutlich bessere Entwicklungschancen besitzen als jene ohne. Die Studierendenzahlen wuchsen in den Hochschulstandorten der ländlichen Regionen in der Vergangenheit sogar etwas stärker als in Raumtypen mit höherem Verdichtungsgrad. Der Braindrain Hochqualifizierter aus ländlichen Regionen wird jedoch nach deren Studium deutlicher als nach dem Schulabschluss, auch deshalb, weil die Qualifikationsprofile der Hochschulen und die Arbeitsplatznachfragen aus den jeweiligen Regionen nur unvollständig zusammenpassen. Im Hinblick auf die zukünftige Entwicklung wird dann die Hypothese vom Bologna-Drain vorgestellt: Demnach könnte die vollzogene Einführung gestufter Bachelor- und Masterstudiengänge dazu führen, dass sich Studienstandorte in unterschiedlichen Regionstypen in ihrer Entwicklung stark unterscheiden und hierbei insbesondere die ländlichen Regionen im Wettbewerb um die besten Köpfe verlieren. Diese These wird explorativ am Beispiel der Justus-Liebig-Universität Gießen beleuchtet, welche als traditionsreiche Universität mit einem großen Fächerspektrum in einer ländlichen Region liegt.

Schlüsselwörter Studienstandortwahl $\cdot$ Bologna-Reform $\cdot$ Mobilität $\cdot$ Bologna-Drain $\cdot$ Ländliche Regionen

Philipp Gareis

Philipp.Gareis@geogr.uni-giessen.de

Prof. Dr. Christian Diller

christian.diller@geogr.uni-giessen.de

Henning Huchthausen

henning.huchthausen@geogr.uni-giessen.de

1 Institut für Geographie, Justus-Liebig-Universität Gießen,

Senckenbergstraße 1, 35390 Gießen, Deutschland 


\title{
Brain Drain and Bologna drain - Spatial implications of the Bologna reform and impacts on rural regions: An analysis on the basis of the example Justus-Liebig-University Giessen
}

\begin{abstract}
This article deals with reasons and spatial distribution patterns of the choice of location of undergraduates and students and the connected spatial impacts on the regional development, especially in rural regions in Germany. First, the international state of research is presented for the choice of location of students on different points of time of their educational path. An analysis of regional statistical data linked to the finding that higher education institutions in rural regions are not able to create the same stock of students as urbanised areas, but rural regions have better chances for economic development in the presence of a higher education institution. The number of students in rural areas with higher education institutions increased even more than in sareas or metropolises. However, the brain drain of highly qualified manpower from rural regions will increase after their years of study. This is caused by a better fitting skill profile in more densely populated areas for highly skilled workforce as well as for the supply of these jobs. Whether these tendencies already start during the transition from the bachelor to master degree will be examined with data from the Justus-Liebig-University Gießen, a university in a rural region. With a view to the future development the hypothesis of the bologna drain is introduced. It implies that the transition from bachelor to master, which did not exist in Germany before the year 2000, locations to study vary a lot in their development and especially rural regions loose in the competition for brilliant minds.
\end{abstract}

Keywords Choice of location for studies $\cdot$ Bologna reform $\cdot$ Mobility $\cdot$ Bologna drain $\cdot$ Rural regions

\section{Einleitung}

Die Rolle der Hochschulen rückt seit Beginn der Transformationsprozesse hin zur Wissensgesellschaft immer stärker in den Fokus der Diskussion zur regionalen Entwicklung (Kujath/Stein 2009). Dieser Artikel stellt den Faktor Studierende in den Vordergrund. In früheren Betrachtungen wurde der regionalökonomische Nutzen von Hochschulen und damit auch ihrer Studierenden noch weitgehend über Nachfrageeffekte definiert. Die Rolle der Studierenden war auf die als Nutzer regionaler Dienstleistungen reduziert (vgl. Fromhold-Eisebith 1992: 26; Bred1/Liefner/Teichert et al. 2014; Glückler/Panitz/Wuttke 2015). Zunehmend wird jetzt aber auch die Frage nach dem Verbleib der Studierenden in der Region nach dem Studium gestellt und wie ihr Qualifikationspotenzial in den Regionen aufgenommen werden kann. Dabei werden sowohl die Chancen des regionalen Wissenstransfers durch Hochschulen als Forschungsressource oder durch Spin-off-Gründungen herausgestellt als auch der positive Effekt des personellen Wissenstransfers durch Hochschulabsolventen. Hierbei stehen ländliche Regionen immer wieder im Fokus einer regionalpolitisch ausgerichteten Hochschulpolitik. Ziel einer solchen Hochschulpolitik war und ist es auch, dem Braindrain, also der Abwanderung potenzieller Hochqualifizierter aus den ländlichen Regionen in die Agglomerationen, entgegenzuwirken (Prenzel 2015: 4f.). So wurden beispielsweise in Bayern in den 1960erund 1970er-Jahren dezidiert Hochschulgründungen in ländlichen Regionen vorangetrieben. Ähnlich baute beispielsweise Brandenburg nach 1990 gezielt Hochschulstandorte in peripheren Landesteilen aus.
Wenn die Absolventenzahlen von Hochschulen und die Qualifikationsstruktur in Regionen miteinander korrelieren (vgl. Falk/Kratz 2009: 64; Jaeger/Kopper 2014), stellen sich folgende Untersuchungsfragen:

- Woher kommen die Schulabsolventen, Studierenden und Hochschulabsolventen?

- Wo verbleiben die Studierenden nach dem Absolvieren ihres Studiums?

- Gibt es Veränderungen der Mobilität und des Verbleibens am Hochschulstandort von Schulabsolventen, Studierenden und Hochschulabsolventen seit Implementierung der Bologna-Reform, insbesondere in ländlichen Regionen, beim Übergang vom Bachelor zum Master?

In diesem Beitrag wird zunächst in Kapitel 2 der internationale Stand der Forschung nach den einzelnen Stationen in der Bildungsbiographie von Studierenden skizziert. Dies ist sinnvoll, weil sich die meisten Untersuchungen nur auf eine Station der Bildungsbiographie konzentrieren, vor allem aber weil die Frage nach den Auswirkungen der Bologna-Reform die Zusammenschau mehrerer Stationen erfordert. Anschließend werden neben deskriptiven Auswertungen von Sekundärstatistiken zur Erklärung von Herkunft und Verbleib der Studierenden in Deutschland mit Hilfe von Studierendendaten aus Gießen (Fallstudie; Kapitel 5) die Veränderungen des Mobilitätsverhaltens durch die Bologna-Reformen dargestellt. Im vierten Kapitel wird die These vom Bologna-Drain eingeführt. Demnach könnte die Einführung gestufter Bachelor- und Master-Studiengänge, die eine weitere, auch räumliche, Wahlentscheidung der Studierenden beinhaltet, dazu führen, dass es räumliche Verschiebungen der Studierendenzahlen an Hochschul- 
standorten in unterschiedlichen Regionstypen gibt und diese sich insbesondere in den ländlichen Regionen in der $\mathrm{Zu}-$ kunft weiter zum Negativen verändern werden. Im Fazit (Kapitel 6) werden erste Handlungsempfehlungen umrissen und weitere Forschungsbedarfe aufgezeigt.

\section{Stand der Forschung: Übergänge in Bildungsbiographien, räumliche Faktoren bei der Studienortwahl, Auswirkungen von Studierenden und Hochqualifizierten auf die regionale Wirtschaftsentwicklung}

Die Forschungsergebnisse der Einflüsse der Studierendenmobilität auf die Regionalentwicklung in unterschiedlichen Raumtypen (vgl. Abbildung 1 für detaillierte Auswertungen) lassen sich nach den einzelnen Phasen bzw. Stationen von Studierendenlaufbahnen sortieren: vom Schulabschluss am Anfang bis zum Übergang ins Berufsleben am Ende und - seit der Einführung gestufter Studiengänge - zusätzlich mit der Station des Übergangs vom Bachelor zum Master in der Mitte.

Die nachfolgende Auswertung des Forschungsstandes bezieht sich vor allem auf Ergebnisse in Deutschland, punktuell sind Untersuchungen für die USA, Großbritannien, die Schweiz und die Niederlande einbezogen. Dies hängt zum einen mit der Verfügbarkeit geeigneter Studien zusammen, zum anderen mit dem Umstand eines in großen Zeiträumen ähnlichen Studiensystems (Schweiz) und mit einem durchgehend zweigeteilten Studiensystem als Vergleichsgröße (USA, Großbritannien, Niederlande). Die Studien sind, geordnet nach ihren Untersuchungsschwerpunkten, in Abbildung 1 aufgeführt. Weitere Querschnittstudien finden im Rahmen der Zusammenfassung des Forschungsstandes im weiteren Verlauf dieses Kapitels Berücksichtigung.

Zunächst sind die Untersuchungen hinsichtlich ihrer gewählten Methodik, ihrer Untersuchungsfragen und der präsentierten Ergebnisse zu unterscheiden. So zeigen sich Unterschiede der Untersuchungsziele der in Abbildung 1 dargestellten Studien, die zum einen den Studieneinstieg und fächerbezogene Unterschiede in den Blick nahmen (Abbildung 1: Pfeil 1) und zum anderen die Verbleibesituation an den Hochschulstandorten betrachteten (Abbildung 1: Pfeile 3 und 4). Beim Übergang von der Schule zur Hochschule (Pfeil 1 in Abbildung 1) zeigt sich, dass die räumliche Nähe zur Hochschule die Studienstandortwahl nach dem Schulabschluss beeinflusst. Dies ist durch verschiedene Studien, insbesondere Schüler- und Studierendenbefragungen, für mehrere Staaten belegt (Montgomery 2002; Falk/Kratz 2009; De Boer/Kolster/Vossensteyn 2010). Dabei spielen diverse Faktoren eine Rolle: Zum einen ist die deutlich niedrigere Quote von Erwerbstätigen mit Hochschulabschluss in ländlichen Räumen, in welchen weniger
Hochschulen bzw. weniger große Hochschulen beheimatet sind, zu berücksichtigen. Zum anderen zeigen die unter dem ersten Pfeil angegebenen Studien die Wirksamkeit von Peer-Effekten, wonach potenzielle Studierende in ihrer Entscheidung für die Studienortwahl vom direkten sozialen und ökonomischen Umfeld beeinflusst werden. Eine weitere Rolle spielen auch emotionale Kosten, denn durch einen Wohnortwechsel müssen Bindungen aufgegeben und erhöhte Transaktionskosten in Kauf genommen werden, da Wohnungs- und Reisekosten am neuen Standort höher sind (Lörz 2008: 429). Nachfolgende Untersuchungen kommen zu interessanten Befunden hinsichtlich räumlicher Faktoren und Motive:

Hachmeister, Harde und Lange (2007) untersuchten beispielsweise mittels einer Onlineumfrage unter deutschen Abiturienten, welche Absichten bezüglich der Studien(standort)wahl bestehen. Die Präferenzen der Studienstandortwahl der befragten Abiturienten unterschieden sich vor allem hinsichtlich der Fachrichtungen. Der Typus des „Heimatgebundenen Hedonisten“ (Hachmeister/Harde/ Lange 2007: 65), der vor allem in den Fachrichtungen Gesundheitswissenschaften/Medizin sowie Sprach- und Kulturwissenschaften, seltener in den Ingenieur- und $\mathrm{Na}$ turwissenschaften vorzufinden war, zeigte erste Unterschiede in Fächerneigungen und Mobilitätsbereitschaft. Jedoch ist hierbei kritisch $\mathrm{zu}$ fragen, ob die von den befragten Abiturienten beabsichtigten Fächer- und Standortwahlen tatsächlich umgesetzt wurden.

Einen valideren Ansatz wählten Falk und Kratz (2009). Sie untersuchten mittels eines Panels die regionale Mobilität bayerischer Hochschulabsolventen. Hierbei fanden sie heraus, dass die Absolventen des Jahrgangs $2006 \mathrm{zu}$ $78 \%$ ihre Hochschulzugangsberechtigung in Bayern erworben hatten und weitere Absolventen hauptsächlich aus den Nachbarbundesländern Hessen und Baden-Württemberg ihr Studium in Bayern absolvierten (Falk/Kratz 2009: 58). Diese Ergebnisse stehen in deutlichem Widerspruch $\mathrm{zu}$ den von Hachmeister, Harde und Lange (2007) aufgezeigten Befragungsergebnissen, sind jedoch aufgrund der Paneldatenerhebung über einen längeren Zeitraum als konsistenter anzusehen. Ebenso ist der starke Rücklauf der Befragung von Falk und Kratz (2009: 56) mit knapp $40 \%$ mittels des Adressmitteilungsverfahrens zu nennen, welcher aussagekräftigere Ergebnisse liefert als vergleichbare Studien. Neben den Unterschieden in der Fächer- und Standortwahl ist in dieser Studie auch die Bindungswirkung der Hochschulregionen untersucht worden. Falk und Kratz (2009) zeigten deutlich, dass die Bindungswirkung der Hochschulstandorte beim Berufseinstieg in Agglomerationsräumen (München, Nürnberg, Augsburg) höher ist als in kleineren Hochschulstandorten (Eichstätt-Ingolstadt, Passau, Bayreuth).

Gestützt werden diese Ergebnisse durch die Untersuchung von Brugger und Wolters (2012). Hier wurde das 


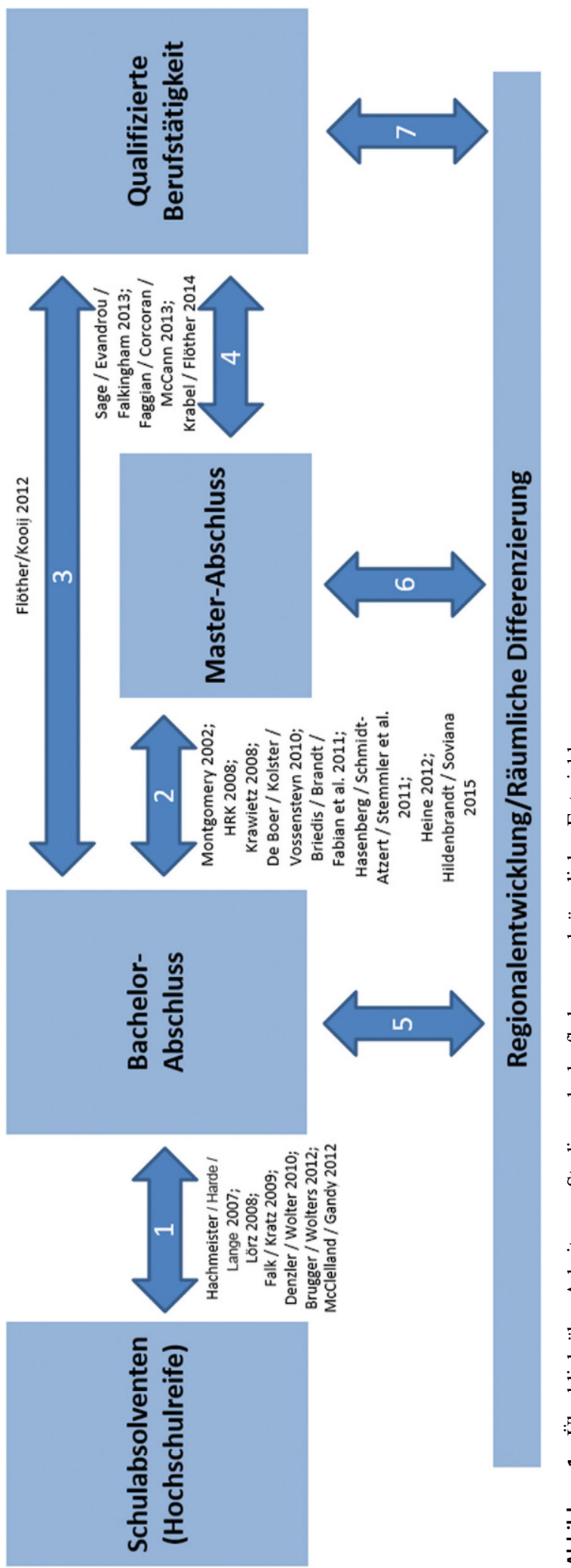

räumliche Verhalten beim Übergang von der Schule zum Studium differenziert nach den deutschen Bundesländern betrachtet. Dabei konnten vor allem deutliche Wanderungsgewinne der Stadtstaaten gegenüber ihren umgebenden Flächenländern festgestellt werden. Krabel und Flöther (2014) untersuchten mit Hilfe einer zweistufigen Analyse auf der Ebene der NUTS-2-Regionen sowohl den Berufseinstieg der Studierenden als auch die Wahrscheinlichkeit, dass der Hochschulabsolvent die Region der Hochschule verlässt, nachdem woanders eine Arbeitsstelle gefunden wurde. Es zeigte sich, dass zwei Drittel der Befragten die Hochschulregion nach dem Abschluss verließen. Bei einer Berücksichtigung der Unterschiede hinsichtlich der Beschäftigungsfähigkeit in der Region ergab sich, dass die wirtschaftlichen Faktoren sowie die Lebensqualität der Hochschulregion sowie die Kontakte der Hochschulabsolventen zu Arbeitgebern vor Ort die wichtigsten Determinanten der regionalen Mobilität beim Eintritt in den Arbeitsmarkt darstellten (Krabel/Flöther 2014).

Jaeger und Kopper (2014) wiesen nach, dass es bezüglich der Mobilität bei städtischen Hochschulorten kaum Unterschiede zwischen Universitäten und Fachhochschulen in den Abwanderungsquoten der Absolventen gab. In ländlichen Regionen zeigte sich jedoch, dass die Passgenauigkeit der Fachhochschulen für den Arbeitsmarkt oftmals eher der regionalen Wirtschaftsstruktur entsprachen als die Ausbildungsangebote von Universitäten. Dies zeichnete sich durch die höhere Bindungswirkung der Fachhochschulstandorte im Vergleich zu Universitätsstandorten aus (Jaeger/Kopper 2014). Ebenso ist diese Feststellung mit den (nicht in Abbildung 1 aufgeführten) Erkenntnissen von Kratz und Lenz (2015) in Verbindung zu sehen, welche ebenfalls die stärkere Bindung der Absolventen der Fachhochschulen in ihrer Hochschulregion beobachteten. Auch die Querschnittstudie von Tarazona (2010) erkennt insgesamt einen deutlich positiven Zusammenhang zwischen hohen Quoten von sozialversicherungspflichtig Beschäftigten mit Abitur oder Hochschulabschluss und der Beschäftigtenentwicklung einer Region.

Beim Blick auf internationale Studien zeichnen sich weitere Merkmale der Bindungswirkung von Hochschulstandorten ab. Die folgenden beiden Untersuchungen, die nicht das zweigeteilte System bzw. die Mobilität als Untersuchungsschwerpunkt, sondern die wirtschaftlichen Auswirkungen als Fokus hatten, stützen somit die Mobilitätsunterschiede zwischen der Mobilität von Universitätsund Fachhochschulabsolventen. Faggian, Corcoran und McCann (2013: 338f.) wiesen für Großbritannien nach, dass mit höherem Bildungsabschluss auch größere Entfernungen von den Absolventen für den Berufseinstieg in Kauf genommen werden. Des Weiteren zeigen die Autoren, dass die Entfernungen zum Ort des Berufseinstigs höher sind, je mehr Prestige die besuchte Hochschule hat 
und je besser die Noten sind. Dies ist laut den Autoren den Spezialisierungen im weiteren Studienverlauf zuzuschreiben. Eine weitere Untersuchung zu Großbritannien von Sage, Evandrou und Falkingham (2013: $748 \mathrm{ff}$.) ergab, dass im Zeitraum der ersten fünf Jahre nach dem Studium, die Zeit einer gewissen Instabilität, fast die Hälfte der Befragten in das Elternhaus zurückkehrte, und von denen wiederum die Hälfte dann auch mehr als ein Jahr dort lebte. Dies könnte auch Einfluss auf die spätere Beschäftigung haben, da Hochschulabsolventen in vielen Fällen die Hochschulregion verlassen und ein Berufseinstieg in der Heimatregion unter den genannten Umständen potenziell wahrscheinlicher wird.

Die indirekten und nicht beeinflussbaren Effekte, die das Mobilitätsverhalten in Bezug auf die Studienstandortwahl beeinflussen, sind ebenso ein wichtiger Aspekt vieler Untersuchungen. Brugger und Wolters (2012) wiesen nicht nur den Zugewinn von Studierenden für Stadtstaaten beim Übergang von der Schule zur Hochschule nach. Sie belegten auch, dass dieser Befund so robust ist, dass er zwar durch andere Effekte wie Studiengebühren oder strukturelle Verbesserungen der Hochschulsysteme überlagert wird, aber dennoch erhalten bleibt. Lörz (2008) zeigte außerdem, dass Studierende mit einer besseren materiellen Grundausstattung und aus sozioökonomisch meist besser gestellten Akademikerhaushalten deutlich freier in der Wahl ihrer Hochschule sein können, weil sie weniger den mit hohen Kosten verbundenen Distanzrestriktionen wie beispielsweise Wohn- und Lebenshaltungskosten vor Ort unterworfen sind. Dies konnte auch für Studierende in der Schweiz nachgewiesen werden (Denzler/Wolter 2010). Differenziert man weiter nach der schulischen Leistung, lässt sich feststellen, dass die besten Maturanden sich in ihren Studienwahlentscheidungen nicht durch die Distanz zur Hochschule beeinflussen ließen (Denzler/Wolter 2010: 694). Ein weiterer Aspekt, der nachgewiesen werden konnte, war, dass die Mobilität besonders bei den jüngeren Studierenden, die direkt im Anschluss an die Schule ein Studium aufgenommen hatten, höher ist (Sage/Evandrou/ Falkingham 2013).

McClelland und Gandy (2012) untersuchten die Wanderungsbewegungen der Studierenden in Großbritannien hinsichtlich von Nachfrage und Angebot an Studienplätzen. Hierbei stellten sie einen wachsenden Trend hin zum Studium an wohnortnahen Hochschulen fest, insbesondere bei Frauen. Die Gründe hierfür lagen vor allem in den im Süden Großbritanniens sehr hohen regionalen Lebenshaltungskosten (Mieten) - eine Entwicklung, die in den letzten Jahren auch in vielen deutschen Ballungsräumen zu beobachten war. Singleton, Wilson und O'Brien (2012) zeigten weitergehend in ihrer regionalstatistischen Analyse des Wanderungsverhaltens von Schulabsolventen zu Hochschulen zunächst, dass die Funktion von Abwanderungswahr- scheinlichkeit und räumlicher Wanderungsentfernung nicht linear, sondern exponentiell verläuft. Zunächst sinkt die Wahrscheinlichkeit mit jedem Kilometer Entfernung zur Hochschule, diese zu besuchen, exponentiell sehr stark, um dann mit zunehmender Entfernung nur noch sehr langsam zu sinken. Das heißt, falls ein Wohnortwechsel aufgrund zu großer Entfernungen zum Heimatort stattfindet, ist eine stark zunehmende Distanz zum Heimatort nur noch ein geringes Hemmnis bei der Studienstandortwahl und der Wohnortwechsel sehr wahrscheinlich durch andere Motive zu erklären (vgl. Hachmeister/Harde/Lange 2007: 91).

Zur Frage nach dem Übergang vom Bachelor zum Master (Pfeil 2 in Abbildung 1) liegen mittlerweile auch für Deutschland einige bundesweite Untersuchungen vor. Diese liefern erste Indizien für das Mobilitätsverhalten von Studierenden unterschiedlicher Fächer und Hochschultypen. Die erste repräsentative Befragung wurde von der Hochschulrektorenkonferenz vorgelegt (HRK 2008). Hier wurden Bestimmungsfaktoren der Mobilität von 5.064 Studierenden ermittelt. $24 \%$ der Befragten in einem Masterstudiengang gaben an, im Studienverlauf mindestens einmal die Hochschule im Inland gewechselt zu haben, weitaus mehr als in den Studiengängen Diplom, Magister und Staatsexamen (HRK 2008: 7). Für die Schnittstelle Bachelor-Master ist das Ziel einer Steigerung der Mobilitätsquoten, wie es in der Bologna-Erklärung (Gemeinsame Erklärung der Europäischen Bildungsminister 1999) das Ziel war, bereits 2008 praktisch erfüllt gewesen. Innerhalb des Bachelorstudiums hingegen wurde sehr selten (1\%) der Standort gewechselt (HRK 2008: 18). Diese geringere Mobilität der Bachelorstudierenden im Verlauf ihres Studiums im Vergleich zu den Diplomstudierenden führte Krawietz (2008: 403) vor allem auf das verschultere Curriculum im Bachelorstudiengang zurück.

Die mobilitätsbedingten Unterschiede zwischen Universitäten und Fachhochschulen sowie auch zwischen Studierenden bestimmter Fächergruppen seit Implementierung der Bologna-Reform sind weitere Betrachtungselemente beim Übergang vom Bachelor auf den Master (vgl. Krawietz 2008). Studierende aus Sprach-, Kultur- und Sportwissenschaften, Rechtswissenschaften sowie Sozial- und Wirtschaftswissenschaften waren sowohl räumlich als auch fachlich deutlich mobiler als Studierende der Natur- und Ingenieur- sowie Forst-, Agrar- und Ernährungswissenschaften. Universitätsstudierende zeigten sich weitergehend deutlich mobiler als Studierende an Fachhochschulen (HRK 2008). Eine weitere Untersuchung zum Übergang vom Bachelor auf den Master wurde für den Studienjahrgang 2009 von Briedis, Brandt, Fabian et al. (2011) vorgelegt. Ein Jahr nach ihrem Bachelorabschluss hatten $77 \%$ der befragten Universitätsstudierenden sowie $54 \%$ der Fachhochschüler ein Masterstudium begonnen, weitere etwa $10 \%$ hatten dies geplant. Hier zeigte sich, 
dass $46 \%$ der Fachhochschüler, jedoch nur $36 \%$ der Universitätsabsolventen die Hochschule für das Masterstudium wechselten (Briedis/Brandt/Fabian et al. 2011: 66). An den Fachhochschulen und in den Naturwissenschaften war der Anteil der Hochschulwechsler geringer als in Geistes-, Rechts- und Wirtschafts-/Sozialwissenschaften sowie zwischen den Universitäten und Fachhochschulen generell. Auch die Übergangsquote vom Bachelor zum Master war an Fachhochschulen geringer als an Universitäten. Zudem wurde das Masterstudium an Universitäten meist direkt im Anschluss aufgenommen (Heine 2012).

Im Hinblick auf die Masterstudierenden (Abbildung 1, Pfeile 2 und 4) liefern die Untersuchungen unterschiedliche Erkenntnisse bezüglich der Motive und des Mobilitätsverhaltens. In der Studie der Hochschulrektorenkonferenz (HRK 2008) konnte mittels einer umfangreichen Umfrage herausgefunden werden, dass von den Masterstudierenden, die einen Studiengangs- und Hochschulwechsel vorgenommen hatten, $90 \%$ das Interesse für den Studienschwerpunkt und $66 \%$ die Verbesserung des fachlichen Niveaus als Grund für einen Standortwechsel nannten. Der Ruf der Hochschule spielte für $46 \%$ eine Rolle, andere Gründe wie geringere Studiengebühren oder familiäre Gründe waren nachrangig (HRK 2008: 21). Heine (2012) untermauerte dies, da er als Gründe für den Hochschulwechsel vor allem die Weiterbildungs- und fachlichen Interessen und das Motiv der Berufschancen (80-90 \% der Befragten gaben diese Motive an) in seiner Studie feststellte. Immerhin $30 \%$ der Bachelorabsolventen gaben an, den Master für weitere berufliche Orientierung nutzen zu wollen (Heine 2012: 33). Das sind verglichen mit den anderen Motiven zwar relativ wenige, dennoch könnten in dieser Gruppe auch jene Wechsler vorhanden sein, die nicht nur wegen einer Unzufriedenheit mit der Studiensituation ihren Standort wechselten, sondern auch wegen besserer Arbeitsmarktperspektiven woanders die ehemalige Hochschulregion verließen. Mit Hilfe multivariater Modelle konnte Krawietz (2008) einen Zusammenhang von sozialer Herkunft, persönlicher Lebenssituation sowie bestimmter Fächergruppen mit der Mobilitätsbereitschaft nachweisen. Im Falle des Bachelor-Master-Wechsels sieht der Autor Vorteile gegenüber dem einstufigen System, welche jedoch auch durch fehlende äquivalente Masterstudiengänge am Ort des Bachelorabschlusses erklärt werden können.

Weitere Studien zeigen bei den Masterstudierenden teils deutliche Unterschiede, widersprechen sich aber teils in ihren Ergebnissen. Die Nähe zum Elternhaus spielte beispielsweise in Marburg eine geringere Rolle für Masterstudierende im Vergleich zu Ergebnissen aus bundesdeutschen Vergleichsstudien (Hasenberg/Schmidt-Atzert/Stemmler et al. 2011). In Gießen zeigte sich für die Agrarwissenschaft jedoch ein vollkommen anderes Bild. Die Nähe zum Heimatort, ein weit reichendes Semesterticket sowie die Woh- nungssituation waren sehr wichtige Aspekte bei der Wahl des Studienortes Gießen bei den befragten Agrarwissenschaftlern im Gegensatz zum Renommee der Hochschule. Die Gießener Erkenntnisse (vgl. Kapitel 5) sind deckungsgleich mit anderen deutschlandweiten Erkenntnissen zur Mobilität der Bachelorstudierenden nach ihrem Abschluss. Gewöhnungseffekte sowie ein Rückzug in Richtung Heimat im Zuge der Aufnahme eines Masterstudiums sind somit weitere Gesichtspunkte, die bei der Betrachtung der Mobilität im zweigeteilten System Beachtung finden müssen.

Neben Studien aus Deutschland liegen weitere Erkenntnisse aus dem Ausland, teilweise schon seit Längerem, vor. Da viele Länder das gestufte System früher als Deutschland eingeführt haben, sind die Studienergebnisse allerdings wegen der Unterschiede zum deutschen Hochschulsystem nur eingeschränkt auf Deutschland übertragbar. Die Motive im Ausland können jedoch weitere Indizien liefern, inwieweit Komponenten wie Heimatnähe oder Berufschancen Mobilitätsmuster verändern können. De Boer, Kolster und Vossensteyn (2010) ermittelten die Motive von niederländischen Studierenden, ihr vierjähriges Bachelorprogramm um ein zweisemestriges Masterprogramm zu erweitern eine Option, die in der Zeit der Befragung allerdings nur $10 \%$ der Studierenden wahrnahmen. Hier überwogen intrinsische Motive (insbesondere fachliches oder allgemeines Bildungsinteresse) mit $71 \%$ die extrinsischen Motive (Einkommensverbesserung, akademischer Grad) mit $17 \%$ deutlich, dazu kamen noch $8 \%$ Lifestyle-Motive, wenn Studierende noch nicht in den Arbeitsmarkt einsteigen wollten (De Boer/Kolster/Vossensteyn 2010: 390). Für ein 6+4System $^{1}$ dürften sich die für das 8+2-System ermittelten Relationen der Motive allerdings stärker zugunsten der extrinsischen Motive verschieben. Für die USA versuchte Montgomery (2002) mit Hilfe eines Nested-Logit-Modells, verschiedene Einflüsse auf die Wahl des Studienorts und der Qualität der besuchten (Graduierten-)Hochschule zu messen. Hierbei zeigte sich ein hochsignifikanter Einfluss der Nähe des Heimatortes auf die Wahl des Studienortes. Ferner fiel auch hier auf, dass Studiengebühren einen sehr geringen Einfluss auf die Studienentscheidung mit sich brachten (Montgomery 2002: 477; vgl. HRK 2008; Brugger/Wolter 2012).

Mobilität beim Einstieg in den Bachelor kann ein erster Indikator für die weitere Mobilität im Verlauf des Studiums bzw. beim Berufseinstieg sein. Lörz (2008) wies nach, dass Studierende, welche den Heimatort verließen, auch im weiteren Verlauf deutlich mobiler sind als Studierende, welche nahe dem Heimatort studieren. Dies wurde unterstützt durch weitere Studien. Für Bayern zeigten beispielsweise die empirischen Untersuchungen von Kratz

\footnotetext{
${ }_{1} 6$ Semester Bachelor + 4 Semester Master bzw. 8 Semester Bachelor +2 Semester Master.
} 
und Lenz (2015), dass die Abwanderungsquoten von zugezogenen Hochschulabsolventen weit höher sind als diejenigen von einheimischen Absolventen. Denzler und Wolter (2010) ermittelten im Rahmen ihrer Absolventenbefragung in der Schweiz, dass die Distanz zur nächstgelegenen Hochschule Entscheidungen für bestimmte Studienfächer signifikant beeinflusst. Auf der einen Seite nimmt die Wahrscheinlichkeit, ein Fach zu studieren, welches nur an einer einzigen Hochschule angeboten wird, mit steigender Distanz zu dieser Hochschule ab. Andererseits wächst die Wahrscheinlichkeit, ein ganz bestimmtes Fach zu studieren, wenn man in der Nähe einer Hochschule wohnt, welche ein eingeschränktes Fächerangebot mit Schwerpunkt auf diesem Fach aufweist.

Zuletzt sind die regionsspezifischen Unterschiede und Auswirkungen zu nennen (Abbildung 1, Pfeile 5, 6 und 7). Flöther und Kooij (2012) untersuchten in ihrer Querschnittstudie nicht ausschließlich die Übergänge von Bachelor zum Master und Berufseinstieg (Abbildung 1, Pfeil 3), sondern auch die regionalen Auswirkungen. Betrachtet man zunächst nur die Ergebnisse dieser Studie zu den Regionen mit Hochschulen, so zeigt sich, dass die Metropolen der einzige Regionstyp sind, in den mehr Hochschulabsolventen ziehen, als aus diesem Studierende an Hochschulen kamen. Anders gesagt: Vor allem in ländlichen Regionen, aber auch in verstädterten Räumen und ansatzweise sogar in den Agglomerationsräumen (außer in den Metropolen) kann auch eine Hochschule den Braindrain in die Metropolen nicht ganz verhindern. Dies liegt vermutlich vor allem an den dort vermehrt vorhandenen adäquaten Arbeitsplätzen. Bezieht man jedoch die Kreise und kreisfreien Städte ohne eigene Hochschule in die Betrachtung mit ein, so wird deutlich, dass das Vorhandensein einer Hochschule einen erheblichen Unterschied ausmacht. Kamen die Studierenden $\mathrm{zu} 58 \%$ aus Regionen ohne Hochschule, so finden nur $36 \%$ von ihnen nach dem Studium in Regionen ohne Hochschule einen Arbeitsplatz (Flöther/Kooij 2012: 75). Im Gesamtbild jedoch gelingt es auch den Hochschulstandorten in ländlichen Regionen, ihr Hochqualifiziertenpotenzial zu halten. Sie verlieren zwar Absolventen an die stärker verdichteten Regionstypen, es gelingt ihnen jedoch rechnerisch, dies zu kompensieren, indem potenzielle Hochqualifizierte aus dem ländlichen Umland der Hochschulen in der Hochschulregion gebunden werden. Absolventen mit einer Herkunft aus dem ländlichen Raum besitzen dennoch im Gegensatz zu Absolventen aus verstädterten Räumen und Agglomerationsräumen häufiger die Bereitschaft, die Hochschulregion zu verlassen. Die Wirtschaftsund Arbeitsmarktstrukturen sowie die Vielfalt des kulturellen Angebots in den Städten sind demnach besser geeignet, um Hochschulabsolventen, insbesondere aus den ländlichen Regionen, an die verstädterten Regionen und Agglomerationsräume zu binden (Kratz/Lenz 2015). Die Entscheidung, ein Studium im ländlichen Raum aufzunehmen, ist zudem häufig von monetären Anreizen, sprich Lebenshaltungskosten, beeinflusst (vgl. Hasenberg/Schmidt-Atzert/Stemmler et al. 2011). Eine weitere wichtige Rolle spielt hierbei auch das Semesterticket, welches die Mobilität im Verlauf des Studiums sichert (vgl. Hasenberg/Schmidt-Atzert/Stemmler et al. 2011; Hildenbrand/Soviana 2015).

Zusammenfassend lassen sich folgende wiederkehrende Tendenzen in den Untersuchungen festhalten: Die Studienstandortwahl von Schülern und Studierenden ist während ihres Studiums sowohl in Deutschland als auch in den vorgestellten Studien aus dem Ausland zunächst stark geprägt von der Nähe zum Heimatort. Jedoch verliert dieser Umstand im Verlauf der Bildungsbiographie immer mehr an Bedeutung (vgl. Flöther/Kooij 2012; Heine 2012). Materielle Grundvoraussetzungen sowie das Studienangebot in der Nähe des Heimatortes beeinflussen die Studienstandortwahl entscheidend (vgl. Denzler/Wolter 2010). Die Studierenden unterscheiden sich in ihrem Mobilitätsverhalten je nach Studienfach signifikant voneinander. So sind Geistesund Sozialwissenschaftler deutlich mobiler als Ingenieure.

Metropolregionen, städtische Regionen und ländliche Regionen weisen im Studienablauf unterschiedlich starke Bindungswirkungen auf die Studierenden auf. Es zeichnete sich in der Vergangenheit $a b$, dass sich Studierende und Absolventen in stärkerem Maße in Metropolen und verstädterten Räumen konzentrieren und somit Divergenztendenzen befördern. Ländliche Räume waren in den vergangenen Jahrzehnten die Verlierer der Akademisierung der Bildung (vgl. Schubert/Kroll 2014; Fritsch/Piontek 2015). Insbesondere ländliche Regionen ohne Hochschulen verlieren im Wettbewerb um die ,besten Köpfe‘, während ländliche Regionen mit Hochschulen größere Chancen haben (Falk/ Kratz 2009). Die Bedeutung der Studierenden ist nicht nur hinsichtlich der Innovationsfähigkeit für die Regionen mit Hochschulen enorm hoch. Auch in Bezug auf die positive wirtschaftliche Entwicklung wirken in den Regionen mit Hochschulen die Studierenden und Absolventen signifikant positiv auf das Bruttoinlandsprodukt und die Arbeitslosenquote (Schubert/Kroll 2014).

Um diese Erkenntnisse genauer im Hinblick auf die veränderten Studienverläufe durch die Bologna-Reform einordnen zu können, ist zunächst ein Überblick über die Situation und Ausstattung der Regionstypen mit Studierenden und Hochqualifizierten in Deutschland sinnvoll. Ebenso sind erste Wanderungsbewegungen und bevorzugte Verbleibsstandorte mittels amtlicher Statistiken zu erfassen, um die ersten beiden Untersuchungsfragen weitergehend beantworten zu können. 


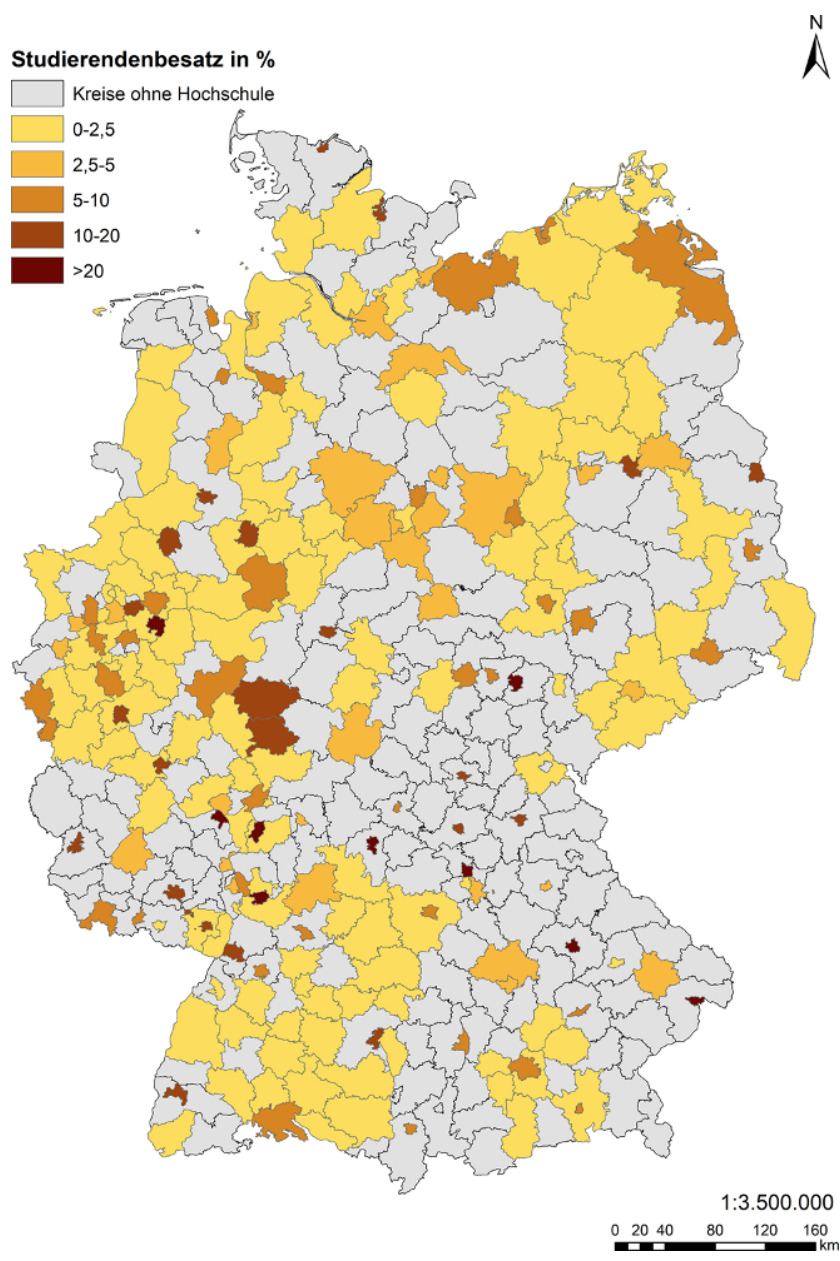

Abbildung 2 Studierendenbesatz gemessen an der Gesamteinwohnerzahl in deutschen Kreisen und kreisfreien Städten 2013. Quelle: Eigene Auswertung, Datengrundlage: Laufende Raumbeobachtung des BBSR (www.inkar.de (13.10.2017); BKG 2017; FDZ 2017)

\section{Regionalstatistische Analysen zur Studierendenentwicklung und -mobilität in Deutschland: raumtypenspezifische Entwicklungen}

Für die Beantwortung der eingangs formulierten Forschungsfragen (vgl. Kapitel 1) ist es erforderlich, die Befunde aus den vorgestellten Studien durch eine regionalstatistische Auswertung zu überprüfen, in welcher die Anteile der Schulabsolventen, Studierenden und Hochqualifizierten an den Einwohnern in Hochschulkreisen sowie allen Kreisen ermittelt werden. Um diese Befunde besser einordnen zu können, werden zunächst die Unterschiede zwischen den Regionen im Studierendenbesatz aufgezeigt. Danach werden die ersten beiden Untersuchungsfragen nach der ersten Gegenüberstellung mit bisherigen Forschungsergebnissen genauer betrachtet: Diese Fragen beziehen sich auf zwei der in Abbildung 1 gezeigten zentralen Stationen in individuellen Bildungsbiographien, nämlich erstens den
Übergang von der Schule zur Hochschule und zweitens den Übergang von der Hochschule in den (hochqualifizierten) Beruf.

Datengrundlage dieser deskriptiven regionalstatistischen Auswertung ist die laufende Raumbeobachtung des Bundesinstituts für Bau-, Stadt- und Raumforschung (BBSR). Betrachtungsebene sind 402 Kreise und kreisfreie Städte, von denen sich im Jahr 2013 in 219 mindestens eine Hochschule mit Studierenden befand. In Anlehnung an Flöther und Kooij (2012) wird hier in Erweiterung der Raumtypisierung des BBSR die kleine Gruppe der deutschen Metropolen (Berlin, Hamburg, München, Köln, Frankfurt am Main) von den anderen Agglomerationsräumen getrennt, dafür kommen die verstädterten Räume und die ländlichen Räume hinzu. Die letzten beiden Kategorien sind beim Thema Hochschulstandorte häufig gemeinsam zu denken. Denn Hochschulen in verstädterten Räumen, hier meist in Mittelstädten oder kleinen Großstädten, fungieren häufig als Bezugspunkte für die daran angrenzenden ländlichen Räume.

Abbildung 2 zeigt den Studierendenbesatz gemessen an den Einwohnerzahlen mit Erstwohnsitz in den deutschen Kreisen und kreisfreien Städten. Dabei wird der Studienort der Studierenden (also die Zahlen der Studierenden an den Hochschulen) betrachtet, nicht ihr Wohnort. Die Karte verdeutlicht die regionalen Unterschiede im Studierendenbesatz. Nur ein kleiner Teil der deutschen Kreise und kreisfreien Städte ist aufgrund fehlender Hochschulen ohne Studierende.

Das räumliche Muster erklärt sich nicht nur durch die Verteilung der Hochschulen, sondern auch durch die unterschiedlichen Zuschnitte der Kreise und kreisfreien Städte in den Bundesländern. Beispielsweise sind viele kreisfreie Städte mit hohem Studierendenbesatz von traditionsreichen und großen Universitäten geprägt. Traditionsreiche und große Universitäten kommen jedoch auch in eher ländlichen Kreisen mit geringer Bevölkerungsdichte vor, wie etwa in Greifswald-Vorpommern. Auch Nordrhein-Westfalen fällt - abgesehen vom Sonderfall des bundesweit rechnerischen Spitzenreiters Hagen mit seiner Fernuniversität - durch einige Kreise und kreisfreie Städte mit besonders hohem Studierendenbesatz auf. Ebenso ist dies in Bayern, Brandenburg und Baden-Württemberg aufgrund der erhöhten Dichte an Hochschulen. In der Flächendarstellung noch evidenter ist jedoch das Bundesland Hessen mit seinen drei Hochschulstandorten in ländlichen Kreisen: Kassel, Gießen und Marburg. Die Metropolen mit den absolut höchsten Studierendenzahlen Berlin, Hamburg, Köln, München und Frankfurt am Main stechen demgegenüber in dieser Abbildung nicht hervor. Deren hohe Studierendenzahl relativiert sich durch die hohe Gesamteinwohnerzahl. Anders stellt sich das Bild allerdings dar, wenn nur der Anteil der Studierenden an allen Einwohnern aus der Altersgruppe der 
Abbildung 3 Unterschiede von Ausbildungsniveaus in allen Kreisen und kreisfreien Städten mit Hochschulen. Quelle: Eigene Auswertung, Datengrundlage: Laufende Raumbeobachtung des BBSR (www. inkar.de (13.10.2017))

\section{Nur Hochschulkreise}

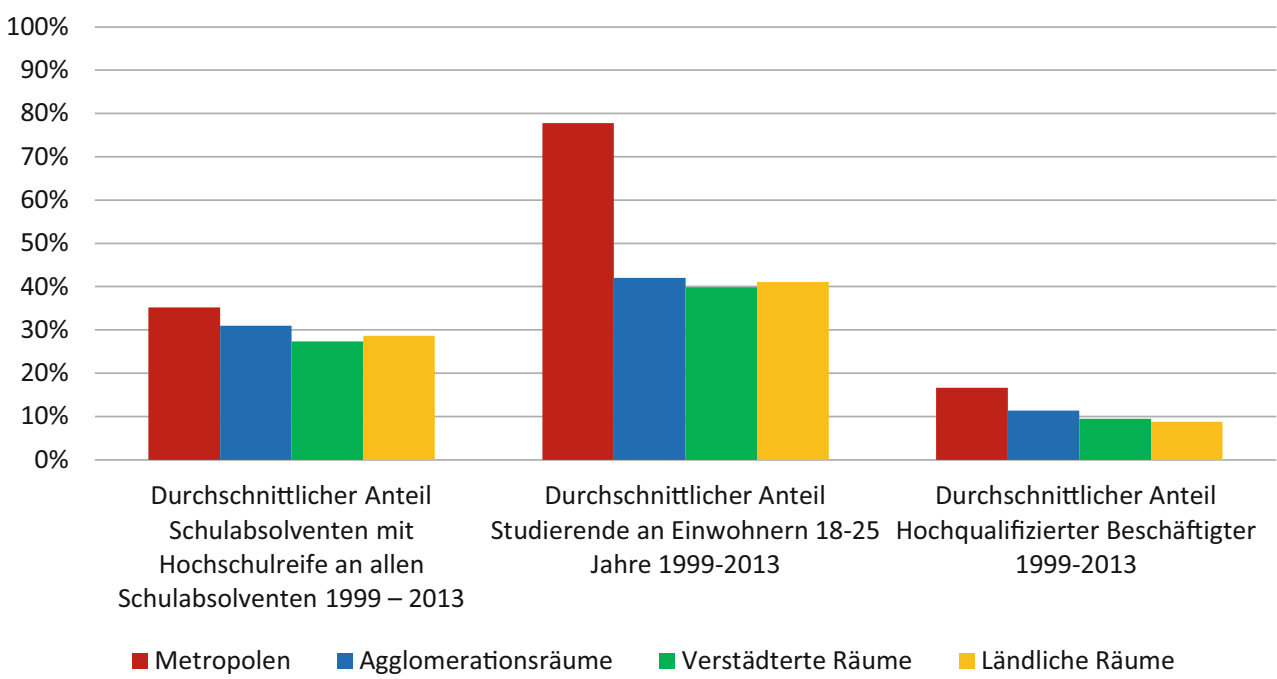

\section{Alle Kreise}

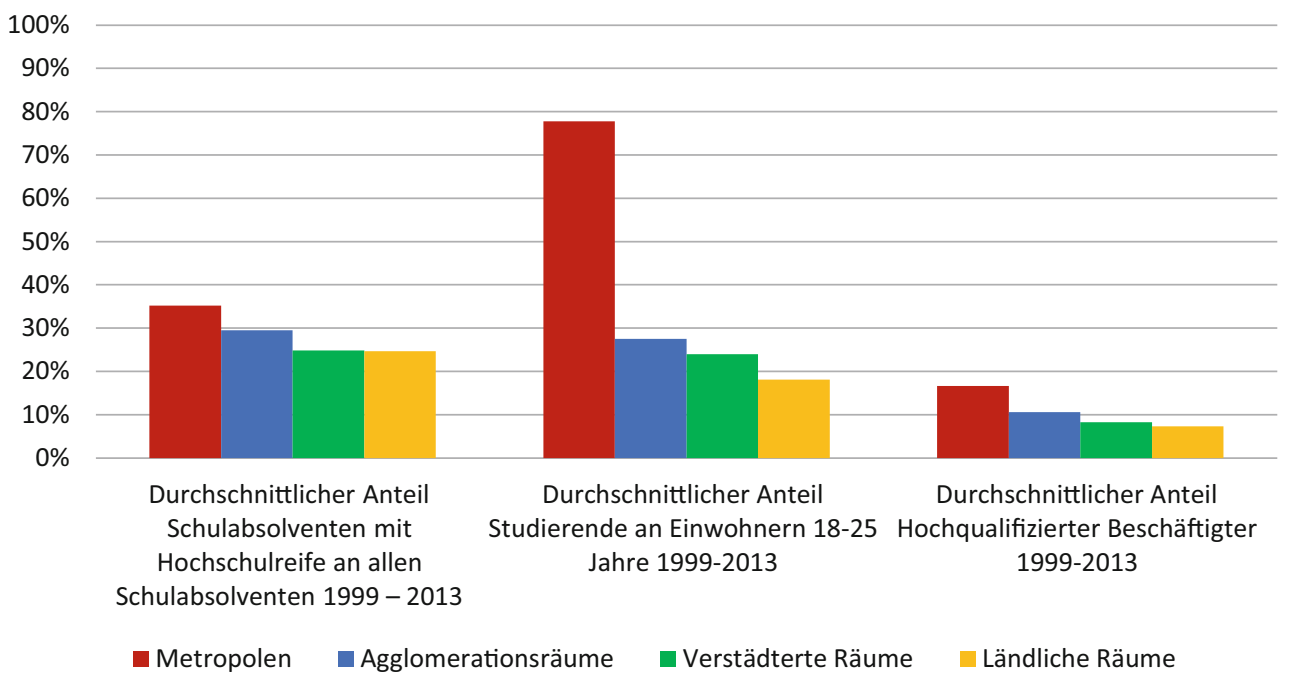

Bildungswanderer zwischen 18-25 Jahren ermittelt wird, welches später noch ausgeführt wird (vgl. Abbildung 3).

Zur Beantwortung der Untersuchungsfragen werden zunächst in allen Kreisen und kreisfreien Städten (obere Reihe in Abbildung 3) der Anteil der Schulabsolventen mit Hochschulreife an allen Schulabsolventen und der Studierendenbesatz an den jungen Einwohnern (der Anteil der Studierenden an der Einwohnerzahl der 18-25-Jährigen) miteinander verglichen. Erkennbar ist, dass sich die Metropolen (5), aber auch die Agglomerationsräume (109) bereits durch den höheren Anteil der Schulabgänger mit Hochschulreife von den anderen Regionstypen abheben. Dem Braindrain durch die Studienortwahl geht noch immer ein Problem des Bildungssystems voraus, dass in ländlichen Regionen (143), aber auch in den verstädterten Räumen (145) die Wahr- scheinlichkeit für einen Schüler, eine Hochschulzugangsberechtigung zu erhalten, geringer ist als in noch stärker verdichteten Räumen. ${ }^{2}$

Vergleicht man nun den Studierendenbesatz bei den jungen Einwohnern mit dem Anteil der Absolventen mit Hochschulreife an allen Schulabsolventen, so fällt vor allem der Sprung bei den Metropolregionen (Berlin, Hamburg, Köln, München und Frankfurt am Main) auf. Während Agglomerationsräume und verstädterte Räume ihr Potenzial halten können, fallen die ländlichen Regionen im Hinblick auf die Betrachtung aller Kreise hier nochmal etwas ab.

Diese Unterschiede werden geringer, wenn nur die Kreise und kreisfreien Städte mit Hochschulstandorten betrach-

\footnotetext{
2 http://www.inkar.de (13.10.2017).
} 
tet werden (untere Reihe in Abbildung 3). Abgesehen von der Gruppe der fünf Metropolen sind hier die Unterschiede zwischen den anderen drei Gruppen sehr gering. Dies bedeutet, dass wenn eine ländliche Region über eine Hochschule verfügt, sie zwar dennoch Schulabgänger verliert (vermutlich vor allem an die Metropolen), sie kann aber Schulabgänger aus umliegenden Kreisen und kreisfreien Städten ohne Hochschulen binden. Rechnerisch haben damit zumindest die ländlichen Kreise mit Hochschulen keinen Braindrain zwischen Schule und Hochschule zu verzeichnen, sie können aber den Braindrain ihres ländlichen Umlandes ohne Hochschule nicht vollständig kompensieren.

Beim Anteil Hochqualifizierter an allen Beschäftigten der Region zeigt sich ein kontinuierliches Stadt-Land-Gefälle. Bemerkenswert ist, dass sich hier Unterschiede zwischen den Regionstypen nicht nivellieren, wenn nur die Regionen mit Hochschulen betrachtet werden. Diese Erkenntnis untermauerten bereits McClelland und Gandy (2012) für Großbritannien, sodass hier tiefergreifende Entscheidungsprozesse von Seiten der Studierenden bzw. Zulassungsbeschränkungen und Auswahlverfahren auf der Angebotsseite der Hochschulen hinter den Verteilungsmustern stecken könnten. Für die ländlichen Regionen wird außerdem deutlich, dass es auch den Kreisen und kreisfreien Städten mit einer Hochschule nicht gelingt, das Potenzial ihrer Hochschulabgänger in der Region zu halten. Ist der Studierendenbesatz in den ländlichen Hochschulstandorten zwar noch höher als in verdichteten Regionen, so fallen sie bei den Hochqualifiziertenanteilen zurück. Dennoch ist es für den Arbeitsmarkt einer ländlichen Region durchaus sinnvoll, über eine Hochschule zu verfügen. In den 83 ländlichen Kreisen ohne Hochschulen liegt der Anteil der Hochqualifizierten bei $6,3 \%$, in den 60 ländlichen Kreisen mit Hochschulen dagegen bei 8,8 \%. Trotzdem kann auch die Existenz einer Hochschule den Braindrain von Hochschulabsolventen aus der ländlichen Region nicht verhindern. Der Anteil hochqualifizierter Beschäftigter ist in den ländlichen Räumen am niedrigsten, und das obwohl die Anteile der Studierenden an den jungen Einwohnern nicht signifikant niedriger sind als in anderen Raumtypen. Dieser Befund aus der regionalstatistischen Analyse bestätigt vor allem die genannten Ergebnisse der bundesweiten Absolventenbefragung (Flöther/Kooij 2012).

\section{Die These des Bologna-Drain}

Die folgenden Überlegungen beziehen sich auf die in $\mathrm{Ab}$ bildung 1 mit Pfeil 2 dargestellte Station der Bildungsbiographie zwischen Bachelor und Master und nehmen damit eine der wichtigsten hochschulpolitischen Änderungen der letzten Jahrzehnte auf. Nach mittlerweile 15 Jahren kann der im Zuge der Bologna-Hochschulreform angestoßene Prozess der Einführung gestufter Studiengänge in Deutschland weitgehend als abgeschlossen bezeichnet werden. Mit dem Wintersemester 2015/2016 waren bundesweit 8.298 Bachelor- und 8.099 Masterstudiengänge an Hochschulen akkreditiert, die große Mehrzahl der Vorgängerstudiengänge Diplom und Magister waren ausgelaufen (HRK 2015: 7). Die Einführung der Bachelor- und Masterstudiengänge wurde im Norden, Nordosten und Westen (Hamburg, Bremen, Schleswig-Holstein, Niedersachsen, Berlin-Brandenburg) Deutschlands deutlich schneller umgesetzt als insbesondere in den süddeutschen Bundesländern (Pietzonka 2014: 24f.).

Eine wichtige Konsequenz der Zweiteilung in Bachelorund Masterstudiengänge ist, dass im Unterschied zu den früheren Diplom- und Magisterstudiengängen, in denen ,in einem Zug 'studiert wurde, das Konzept gestufter Studiengänge eine systematische Zäsur zwischen den beiden Studienabschlüssen vorsieht. Die sechs- bis achtsemestrigen Bachelorstudiengänge sind bereits berufsqualifizierend angelegt, auf diesen bauen in der Regel zwei- bis viersemestrige Masterstudiengänge auf. In den meisten Fächern gilt dabei allerdings faktisch erst der Masterabschluss als eine ausreichende Voraussetzung für den Berufseinstieg (HRK 2015: 8). Das Hauptziel der Bologna-Reform ist die Schaffung eines einheitlichen europäischen Hochschulraums. Neben der stärkeren Praxisnähe und verbesserten Berufsfähigkeit, der Durchlässigkeit der Hochschulen untereinander und zum Arbeitsmarkt, größerer Sozialgerechtigkeit beim Hochschulzugang, der Stärkung der Wettbewerbsfähigkeit und der Verkürzung der Studienzeiten ist die Erhöhung der Studierendenmobilität eines der zentralen Ziele der Reform (Konegen-Grenier 2012: 10 ff.; Pietzonka 2014: 24f.).

Wenngleich Bachelor und Master mehrheitlich direkt nacheinander studiert werden, so hat die Bologna-Reform diesen systematischen Cut zwischen Bachelor- und Masterstudiengang nicht nur mit dem Ziel der stärkeren Ausdifferenzierung von Arbeitsmarktqualifikationen und weiterführenden wissenschaftlichen Qualifikationen eingeführt. Ziel war es auch, die Mobilität innerhalb und außerhalb der eigenen Grenzen zu erhöhen. Dazu wird den Studierenden im Alter von im Regelfall 21/22 Jahren eine systematische Zäsur ihrer Biographie eingeräumt, die es bislang erst am Ende eines Magister- oder Diplomstudienganges gab. Die in Kapitel 2 dargelegten Untersuchungen zeigen, dass das Ziel der gesteigerten Mobilität der Studierenden zwischen Bachelor und Master insgesamt durchaus als erreicht angesehen werden kann.

Die These vom Bologna-Drain besagt nun, dass die Umstellung auf gestufte Studiengänge zu Lasten der Hochschulstandorte in weniger verdichteten Regionen geht, weil die Abwanderung aus der Heimatregion, die vor der Bologna-Reform erst nach dem Studium arbeitsplatzorientiert 
Abbildung 4 Herkunft der Studienanfänger an der Justus-Liebig-Universität Gießen im Zeitraum Wintersemester 1997/1998 bis Sommersemester 2016 nach Kreisen (in \%). Quelle: Eigene Darstellung, BKG 2017, Daten des Studierendensekretariats Gießen 2017

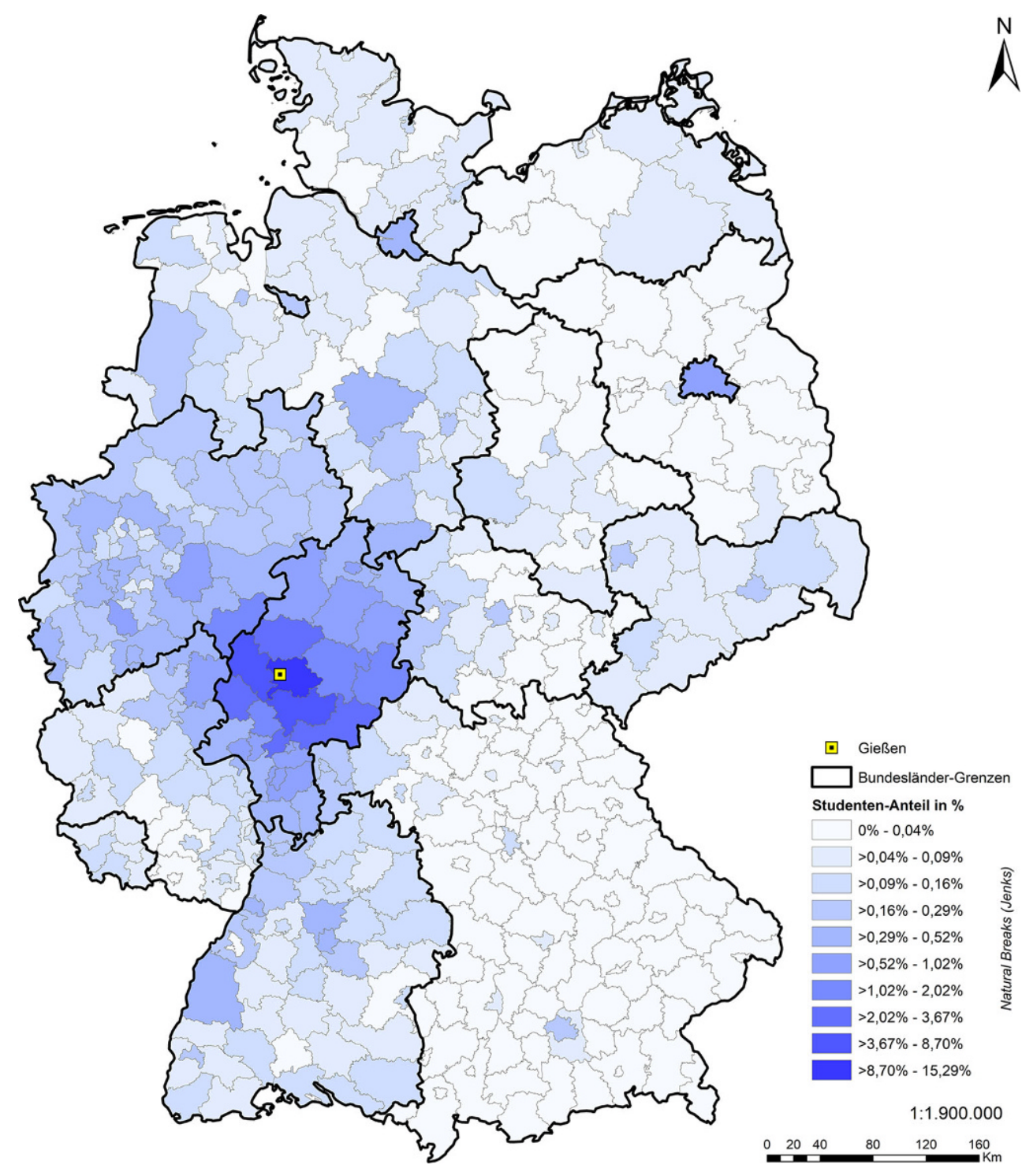

erfolgte, nun von Studierenden zum Teil bereits früher vollzogen wird: beim Übergang vom Bachelor zum Master. Von dem durch die Bologna-Reform beförderten veränderten Studienstandortwahlverhalten, insbesondere im Hinblick auf die Masterstudiengänge, profitieren daher vor allem Agglomerationen, und hier vor allem die Metropolen. Zum ersten können hier die Hochschulen ein breites Portfolio spezialisierter Masterstudiengänge anbieten, zum zweiten haben hier die Absolventen nach dem Studium bessere Arbeitsmarktchancen und zum dritten sind die meisten Großstädte attraktive Lebensorte. Es scheint neben den Studienprofilen auch eine Präferenz nach einem ,Tapetenwechsel` zu geben, die vor allem große Städte attraktiv macht. Die Hochschulstandorte in ländlichen Regionen könnten dagegen einen größeren Teil ihrer potenziellen Hochqualifizierten nicht erst nach dem Studium verlieren, sondern bereits vorher: beim Übergang vom Bachelor zum Master.
Um insbesondere die Situation in den ländlichen Räumen bezüglich des Wanderungsverhaltens der Studierenden im Verlauf der Bildungsbiographie beurteilen zu können, ist es notwendig, Individualdaten zu betrachten. Diese werden im folgenden Kapitel anhand des Fallbeispiels der Justus-Liebig-Universität Gießen analysiert. Dies ist ein erster explorativer Versuch, die Frage des Bologna-Drain beim Übergang vom Bachelor zum Master in ländlichen Regionen zu erfassen.

\section{Fallstudie Justus-Liebig-Universität Gießen}

Die Universitätsstadt Gießen zeichnet sich durch den höchsten Studierendenbesatz in Deutschland aus. Neben der Justus-Liebig-Universität mit knapp 27.000 Studierenden 


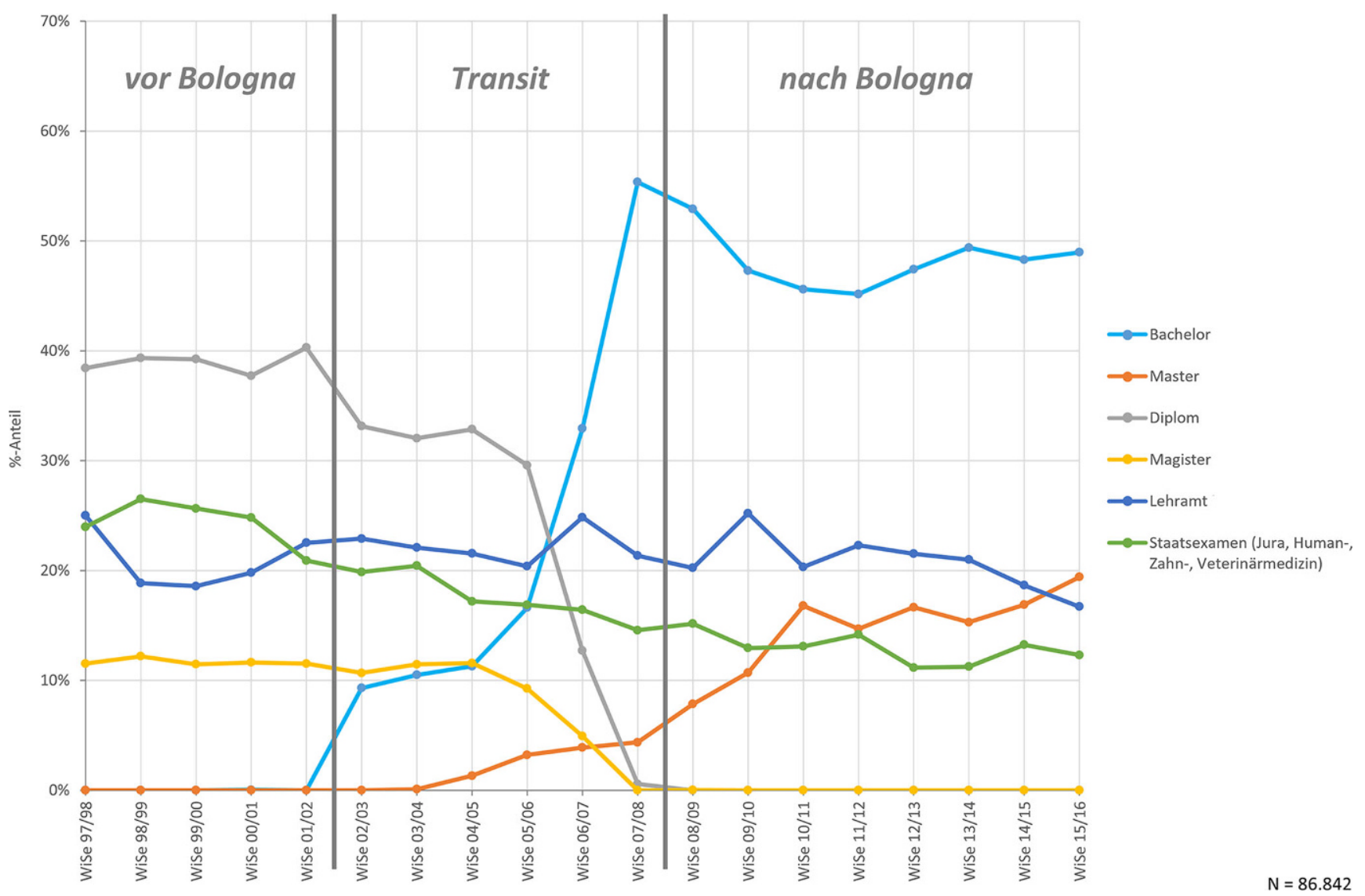

Abbildung 5 Prozentualer Anteil der Studierenden nach Abschlussarten an der Justus-Liebig-Universität Gießen im analysierten Zeitraum Wintersemester 1997/1998 bis Wintersemester 2015/2016. Quelle: Eigene Darstellung, Daten des Studierendensekretariats Gießen 2017

prägt auch die Technische Hochschule Mittelhessen mit etwa 10.000 Studierenden am Campus in Gießen das Stadtbild. ${ }^{3}$ Bezogen auf die knapp 83.000 Einwohner ergibt sich somit ein Studierendenbesatz von knapp $45 \%$. Dieser in Deutschland höchste Wert zeigt die enorme Bedeutung der Gießener Hochschulen für die Stadt, das direkte Umland und die Region Mittelhessen.

Da Individualdaten zum Mobilitätsverhalten von Studierenden auf bundesdeutscher Ebene nicht vorhanden sind, werden hier die Daten des Studierendensekretariats der Justus-Liebig-Universität Gießen ausgewertet. Diese haben den Vorteil, über einen langen Zeitraum verfügbar zu sein (Wintersemester 1997/1998 bis Sommersemester 2016) und die nötigen Informationen zur Herkunft der Studienbeginner sowie dem Verbleib im Verlauf der Hochschulbiographie aufgrund unterschiedlicher Kennzeichnungen (Neueinschreibung, Ersteinschreibung, Rückmeldung) zu besitzen. Dieser Vorteil gegenüber Daten der Forschungsdatenzentren ist in dieser Untersuchung ausschlaggebend, um die Berechnung der Entfernungen vom Ort der Hochschul-

3 Vgl. https://www.giessen.de/Wirtschaft/Wirtschaftsstandort/Zahlen_ und_Fakten/ (04.10.2017). zugangsberechtigung zur Hochschule möglichst genau zu ermitteln. Die Daten liegen anonymisiert als Kreismittelpunkte vor. Mittels google maps application programming interfaces (API) als Programmierschnittstelle wurden Entfernungen als Straßenkilometer berechnet, da diese die reale Entfernung eher wiedergeben als Luftlinienkilometer und somit reale Barrieren aufgrund schlechter Erreichbarkeit von Gießen besser abbilden. ${ }^{4}$

Betrachtet man zunächst die Herkunftsorte der Studierenden seit dem Wintersemester 1997/1998 in Abbildung 4, so erkennt man die starke regionale Verankerung der Justus-Liebig-Universität Gießen. Darüber hinaus ist aber auch das Anziehungspotenzial der Universität in den an Hessen angrenzenden Bundesländern (Thüringen, Nordrhein-Westfalen, Rheinland-Pfalz) sowie in den Stadtstaaten noch als relativ stark einzuschätzen. Bayern bildet hierbei eine Ausnahme.

Die Betrachtung der Studierendenzahlen verschiedener Studienabschlussarten im analysierten Zeitraum an der Justus-Liebig-Universität im Wintersemester lässt weitere Aufschlüsse zu über die räumliche Anziehungswirkung der

4 Vgl. https://www.doogal.co.uk/DrivingDistances.php (04.10.2017). 


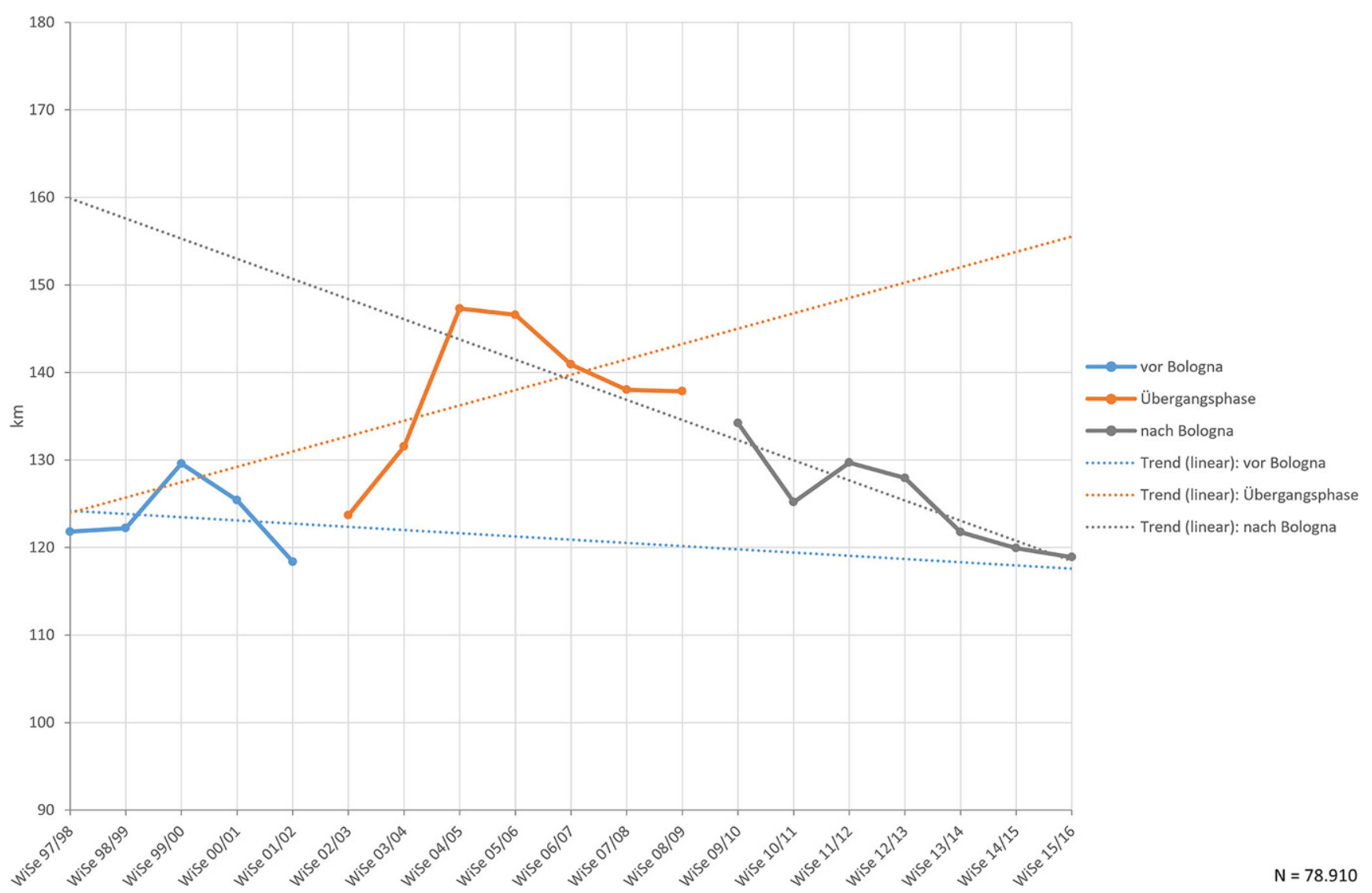

Abbildung 6 Entwicklung der Durchschnittsentfernungen der Gießener Studienanfänger im analysierten Zeitraum Wintersemester 1997/1998 bis Wintersemester 2015/2016. Quelle: Eigene Darstellung, Daten des Studierendensekretariats Gießen 2017

Tabelle 1 Entfernung der Studierenden in den Wirtschaftswissenschaften zwischen dem Ort der Hochschulzugangsberechtigung und der JustusLiebig-Universität Gießen

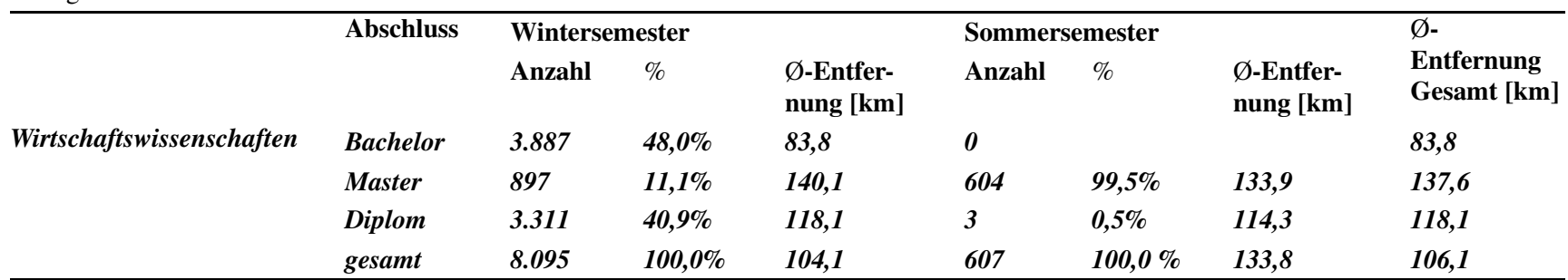

Quelle: Eigene Darstellung, Daten des Studierendensekretariats Gießen 2017

Universität. Dies dient zum einen der Einschätzung der verschiedenen Phasen, die die Hochschule seit Implementierung der Bologna-Reform durchlaufen hat (Vor-Bologna-, Transit- und Nach-Bologna-Phase). Zum anderen wird hierbei deutlich, dass der Anstieg der Masterstudienanfänger im Vergleich zu den Bacheloranfängern deutlich weniger stark ausfiel (vgl. Abbildung 5). Dies kann der Zulassungsbeschränkung der Gießener Masterstudiengänge sowie den Limitationen der Studienplätze geschuldet sein. Aber auch die mangelnde Attraktivität des Standortes als Hochschule im ländlichen Raum könnte eine Ursache der weniger stark nachgefragten Masterstudiengänge sein.
Betrachtet man hierzu die Straßenentfernung der Studienanfänger zum Zentroid des Kreises, in welchem die Hochschulzugangsberechtigung erlangt wurde, zeigt sich, dass es über die Jahre große Unterschiede gegeben hat (vgl. Abbildung 6). Im Verlauf der Umsetzung der Bologna-Reform veränderte sich die Herkunftsentfernung der in Gießen Studierenden deutlich. Während die Vor- und Nach-Bologna-Phase relativ ähnliche Entfernungen aufweisen, ist in der Transitionsphase ein deutlicher Zuwachs bei den Entfernungen der Studienanfänger zu verzeichnen. Dies könnte dem Umstand geschuldet sein, dass zunächst neuartige Studiengänge in Deutschland, also auch in Gießen, entstanden und diese eine hohe Anziehungskraft auch auf weiter 
entfernt lebende potenzielle Studienanfänger ausübten. Der schnelle Studienabschluss des Bachelors selbst, der in Gießen bei sämtlichen Studiengängen mit sechs Semestern Regelstudienzeit eingeführt wurde, könnte ein Grund gewesen sein, der potenzielle Studierende anzog. Andererseits könnten jedoch auch die länger bestehenden Diplomstudiengänge an der Universität ein Grund gewesen sein, verlässliche und anerkannte Abschlüsse zu erwerben und somit diesen Ort als Studienstandort zu wählen. Während der Transitionsphase und zu Beginn der Nach-Bologna-Phase gleicht sich die Entfernung der Studierenden erneut dem Vor-Bologna-Stand an.

Variationen hinsichtlich der Bologna-Reform in einzelnen Fächern lassen sich aufgrund der höchsten Fallzahlen am ehesten mit der laut Heine (2012) (vgl. Kapitel 2) sehr mobilen Gruppe der Betriebs- und Volkswirte nachvollziehen. Ein Vorteil der Betrachtung der wirtschaftswissenschaftlichen Studiengänge liegt in ihrer relativ starken räumlichen Ubiquität sowohl generell in Deutschland als auch im direkten Umland von Gießen (Marburg, Kassel, Frankfurt am Main sowie an der Technischen Hochschule Mittelhessen in Gießen). Somit ist von einer bewussten Entscheidung für den Gießener Studiengang Betriebswirtschaftslehre/Volkswirtschaftslehre von Seiten der Studierenden auszugehen und weniger von einer aufgrund schlechterer finanzieller Ausstattung auf Seiten der Studierenden getroffenen Entscheidung.

Die diversen Abschlussarten in den Wirtschaftswissenschaften, welche im Zeitverlauf in Gießen präsent waren, lassen sich mit Hilfe von Tabelle 1 nachvollziehen. Der Untersuchungszeitraum ist hier erneut vom Wintersemester 1997/1998 bis zum Sommersemester 2016 gewählt, wobei die Studierenden nach ihrem Einschreibungssemester nach Wintersemester und Sommersemester zusammengeführt wurden. Somit gab es seit dem Wintersemester 1997/1998 8.095 Studierende mit Studienbeginn im Wintersemester sowie 607 Studierende mit Studienbeginn im Sommersemester seit dem Sommersemester 1998. Hierbei fällt auf, dass zum einen die Bachelorstudierenden ihre Hochschulzugangsberechtigung räumlich deutlich näher an der JustusLiebig-Universität Gießen erworben und somit dort ihren Lebensmittelpunkt vor dem Studium hatten, andererseits die Masterstudierenden der wirtschaftswissenschaftlichen Studiengänge weiter von Gießen entfernt kamen als die Diplomstudierenden vor der Bologna-Reform. Weitergehend zeigt sich, dass die Studierendenzahlen im Master bei Weitem nicht die Übergangsquote der deutschen Universitäten von $77 \%$ erreichten, sondern weniger als $39 \%$ der Bachelorstudierenden der Wirtschaftswissenschaften auch ihren Masterstudiengang in Gießen beginnen. Ebenso ist der Anteil der Masterstudierenden in den Wirtschaftswissenschaften von nur $24 \%$ zu erwähnen (Studierendensekretariat Gießen 2017). Dies könnte zum einen mit der Studienplatzvergabe zusammenhängen, zum anderen aber auch mit geringen Rückmeldequoten Gießener Studierender im Master sowie geringer Anziehungskraft auf potenzielle Masterstudierende deutschlandweit.

Die oben dargestellten Erkenntnisse lassen auf eine starke Veränderung der Studiensituation im Zeitverlauf schlieBen. Trotz des starken Wachstums der Neueinschreibungen vom Wintersemester 1998/1999 mit knapp 2.600 Studienanfängern hin zu mehr als 6.200 Studienanfängern im Wintersemester 2014/2015 ist dies nicht mit einer steigenden Anziehungskraft der Justus-Liebig-Universität Gießen im weiteren Umland der Hochschule zu erklären, sondern vielmehr mit ihrer steigenden Akademisierung und dadurch stärkeren Anziehungskraft, vor allem auf Bachelorstudierende aus dem direkten Gießener Umland (vgl. Studierendensekretariat Gießen 2017). Dieser Umstand lässt sich jedoch nicht auf die Masterstudiengänge der Justus-LiebigUniversität übertragen. Diese besitzen zwar eine erhöhte Anziehungskraft auf Studierende von außerhalb des GieBener Umlandes, was sich in den höchsten Entfernungen zum Heimatort im Vergleich aller Studienabschlüsse niederschlägt. Jedoch bleiben im Vergleich zum bundesdeutschen Durchschnitt weniger Bachelorstudierende nach ihrem Abschluss in Gießen, sondern sie finden in der Region oder woanders eine erste Arbeitsstelle oder schließen das Studium zu einem gewissen Prozentsatz gar nicht erst ab. Dieser Braindrain, welcher in Gießen am Beispiel der wirtschaftswissenschaftlichen Studiengänge in Tabelle 1 offensichtlich geworden ist, könnte weitreichende Folgen für ähnlich strukturierte Hochschulen im ländlichen Raum sowie deren Regionen besitzen.

\section{Fazit, Handlungs- und Forschungsbedarf}

Die eigene Auswertung der Regionaldaten zu Schulabschlüssen, Studierenden und hochqualifizierten Beschäftigten bestätigte zunächst die Vermutung, dass die Existenz einer Hochschule Agglomerationsnachteile von ländlichen Regionen bei der Attrahierung qualifizierter Beschäftigter zwar nicht vollständig, aber immerhin zum Teil ausgleichen kann. Zugleich wurde in methodischer Hinsicht bestätigt, dass bei diesem Thema eine Verknüpfung der Untersuchung mit einer regionalstatistischen Auswertung und mit Primärerhebungen auf der Individualebene zielführend ist. Demgegenüber zeigt die Auswertung des internationalen Standes der Forschung zur Frage nach dem Wanderungsverhalten von Studierenden und der Regionalentwicklung Unterschiede, die auch auf die nationalen Bildungssysteme und Faktoren wie Wohnungsmärkte zurückzuführen sind. Es lassen sich einige Gemeinsamkeiten feststellen: Beim Übergang von der Schule zum Hochschulstudium wird zwischen den Faktoren der Fächer und der Nähe der 
Hochschule zum Heimatort abgewogen. Studierende aus einkommensstärkeren Schichten sind hier deutlich mobiler. Den Hochschulen in den ländlichen Regionen gelingt es aber durchaus in weiten Teilen, das Potenzial ihres Hinterlandes an sich zu binden. Die Untersuchungen vom Übergang der Hochschule in den Beruf zeigen vor allem, dass die Hochschulen in ländlichen Regionen ihre dort qualifizierten Studierenden nur in dem Maße halten können, wie auch hochwertige Arbeitsplätze zur Verfügung stehen. Der Braindrain ist hier stärker als nach dem Schulabschluss und nach Teilstationen des Studiums.

Die in Deutschland vor allem in den 1960er Jahren in Westdeutschland und in den 1990er-Jahren gesamtdeutsch verfolgte Politik der Hochschulentwicklung in ländlichen Regionen und deren benachbarten verstädterten Regionen kann also insofern als erfolgreich bezeichnet werden, als sie in vielen Regionen immerhin zumindest dazu führte, dass der Braindrain in der Bildungs- und Berufsbiographie zeitlich verzögert werden konnte. Im vergangenen Jahrzehnt haben sich die Studierendenzahlen in ländlichen Regionen noch positiver entwickelt als in den anderen Regionstypen. Vor allem bei statusniedrigeren Studierendengruppen sind die Nähe zum Elternhaus und die geringeren Lebenshaltungskosten nach dem Studienangebot die wichtigsten Gründe, eine Hochschule in ländlicheren Regionen zu wählen. Das Problem des Braindrain für ländliche Regionen stellt sich eher nach dem (ersten) Studienabschluss. Hier verfügen die ländlichen Regionen oft nicht über das passende Arbeitsplatzangebot für die Studierenden - zum einen, weil insgesamt weniger hochqualifizierte Arbeitsplätze angeboten werden. Zum anderen deutet die Untersuchung von Jaeger/Kopper (2014) darauf hin, dass es auch am fachlichen ,Mismatch', vor allem der an Hochschulen erzeugten hohen Qualifikationen mit den in der Region real nachgefragten Qualifikationen liegen kann, wenn Studierende nach dem Studium die ländliche Region verlassen müssen. Hervorzuheben ist allerdings die positive Rolle der Fachhochschulen (Kratz/Lenz 2015). Ihnen gelingt es besser als den Universitäten, auch die Absolventen bereitzustellen, die zum nachgefragten Hochqualifiziertenprofil der Regionen passen. Damit haben die Fachhochschulen vor allem in den ländlichen Regionen nicht nur aufgrund ihrer eigenen Beschäftigten, sondern auch in der Rolle als Ausbilder eine hohe regionalökonomische Bedeutung.

Für den Übergang vom Bachelor zum Master zeigen die Untersuchungen für die Justus-Liebig-Universität GieBen eine recht hohe Mobilität. Zur Frage des möglichen Bologna-Drain, wonach diese verstärkte Mobilität eher zu Lasten der ländlichen Räume geht, lassen sich noch keine validen Befunde festhalten. Der Umstellungsprozess ist noch nicht lange genug vollzogen, um stabile Muster eines veränderten Wanderungsverhaltens zu erkennen. Zudem sind verlässliche Informationen nur durch die Auswer- tung von Individualdaten möglich, die nicht flächendeckend bundesweit, sondern nur für einzelne Regionen vorliegen. Die ersten Implikationen der Vollerhebung in der Fallstudie Gießen zeigen jedoch, dass es im zeitlichen Verlauf deutliche Änderungen seit der Einführung der Bologna-Reform gegeben hat. Diese gewisse zeitliche Koinzidenz des Umstellungsgrades auf Bachelor/Master mit einem leichten Abschwächen des Aufholens der Hochschulen in den ländlichen Räumen in den letzten Jahren ist zwar auch in Gesamtdeutschland zu erkennen gewesen. Jedoch muss für eine generalisierbare und aussagekräftige Untersuchung der bisherige Datenbestand gesichtet, aufbereitet und bestmöglich quantitativ untersucht werden. Die Beantwortung der Frage, ob der Bologna-Drain in ländlichen Regionen flächendeckend existiert, bedarf weiterer vertiefender Untersuchungen anstelle der bloßen Betrachtung der regionalen Studierendenzahlen. Paneldatenuntersuchungen wie bei Falk/Kratz (2009) oder aber die Betrachtung von Individualdaten, wie in dieser Arbeit vorgelegt, scheinen hierbei die bestmöglichen Untersuchungsansätze zu sein. Insbesondere die Auswirkungen bestimmter Fächer sowie die Nähe $\mathrm{zu}$ anderen Hochschulstandorten sowie Übergangsquoten von der Schule zur Hochschule sind hierbei in Zukunft zu berücksichtigen.

Unabhängig davon sind die Aufgaben für die staatliche, regionale und kommunale Politik für Hochschulstandorte in ländlichen Regionen deutlich. Die wichtigste Aufgabe liegt in der Schnittstelle von Regional- und Hochschulpolitik und in der intensiveren Verflechtung von universitärem Lehrangebot und regionaler Wirtschaft, um die Bindung der Hochqualifizierten an die Region auch nach dem Studium zu stärken. In der Stadtentwicklungspolitik ist die Bereitstellung preisgünstigen Wohnraums für Studierende ein Dauerthema, was in den letzten Jahren sogar in einigen Zentren in ländlichen Räumen an Brisanz gewonnen hat. Auch wenn die ländlichen Räume immer noch erhebliche Lebenshaltungskostenvorteile gegenüber verdichteteren Regionen aufweisen, scheint sich die Verbleibesituation in der Bildungs- und Erwerbsbiographie weiter zu städtischeren Regionen zu verschieben. Dazu muss es in vielen Fällen zur Verbesserung der sonstigen Infrastruktur und der Lebensqualität für Studierende kommen.

\section{Literatur}

BKG - Bundesamt für Kartographie und Geodäsie (2017): Verwaltungsgebiete 1:1.000 000. http://www.geodatenzentrum.de/ geodaten/gdz_rahmen.gdz_div?gdz_spr=deu\&gdz_akt_zeile $=5 \&$ gdz_anz_zeile $=1 \& g d z \_u n t \_z e i l e=18 \& g d z \_u s e r \_i d=0 \# d o k$ (14.04.2018).

Bredl, S.; Liefner, I.; Teichert, C.; Winker, P. (2014): Effekte der Hochschulen am Standort Gießen aus regionalökonomischer Sicht. Marburg. = Joint Discussion Paper Series in Economics by the Universities of Aachen, Gießen, Göttingen, Kassel, Marburg, Siegen 33-2014. 
Briedis, K.; Brandt, G.; Fabian, G.; Rehn, T. (2011): Bachelorabsolventen im Fokus. In: Briedis, K.; Heine, C.; Konegen-Grenier, C.; Schröder, A.-K. (Hrsg.): Mit dem Bachelor in den Beruf. Arbeitsmarktbefähigung und -akzeptanz von Bachelorstudierenden und -absolventen. Essen, 53-82.

Brugger, P.; Wolters, M. (2012): Von der Hochschulreife zum Studienabschluss. In: Wirtschaft und Statistik 8, 655-664.

De Boer, H. F.; Kolster, R.; Vossensteyn, H. (2010): Motives Underlying Bachelors-Masters Transitions: The Case of Dutch Degree Stackers. In: Higher Education Policy 23, 3, 381-396. doi: 10.1057/hep. 2010.14

Denzler, S.; Wolter, S. C. (2010): Der Einfluss des lokalen Hochschulangebots auf die Studienwahl. In: Zeitschrift für Erziehungswissenschaft 13, 4, 683-706. doi: 10.1007/s11618-010-0143-6

Faggian, A.; Corcoran, J.; McCann, P. (2013): Modelling geographical graduate job search using circular statistics. In: Papers in Regional Science 92, 2, 329-343. doi: 10.1111/pirs. 12026

Falk, S.; Kratz, F. (2009): Regionale Mobilität von Hochschulabsolventen beim Berufseinstieg. In: Beiträge zur Hochschulforschung $31,3,52-67$

FDZ - Forschungsdatenzentren der Statistischen Ämter des Bundes und der Länder (2017): Statistik der Studenten 1995-2014. Düsseldorf.

Flöther, C.; Kooij, R. (2012): Hochschulen als Faktoren im regionalen Standortwettbewerb. (K)eine Gewinner-Verlierer-Story? In: Die Hochschule - Journal für Wissenschaft und Bildung 21, 2, 65-80.

Fritsch, M.; Piontek, M. (2015): Die Hochschullandschaft im demographischen Wandel - Entwicklungstrends und Handlungsalternativen. In: Raumforschung und Raumordnung 73, 5, 357-368. doi: 10.1007/s13147-015-0364-5

Fromhold-Eisebith, M. (1992): Wissenschaft und Forschung als regionalwirtschaftliches Potential? Das Beispiel von Rheinisch-Westfälischer Technischer Hochschule und Region Aachen. Aachen. = Informationen und Materialien zur Geographie der Euregio MaasRhein, Beiheft 4.

Gemeinsame Erklärung der Europäischen Bildungsminister (1999): Der Europäische Hochschulraum. Bologna. https://www.bmbf. de/files/bologna_deu.pdf (12.01.2018).

Glückler, J.; Panitz, R.; Wuttke, C. (2015): Die wirtschaftliche Wirkung der Universitäten im Land Baden-Württemberg. In: Raumforschung und Raumordnung 73, 5, 327-342. https://doi.org/10. 1007/s13147-015-0360-9

Hachmeister, C.-D.; Harde, M. E.; Lange, M. F. (2007): Einflussfaktoren der Studienentscheidung. Eine empirische Studie von CHE und EINSTIEG. Gütersloh. = CHE Arbeitspapier 95.

Hasenberg, S.; Schmidt-Atzert, L.; Stemmler, G.; Kohlhaas, G. (2011): Empirische Erkenntnisse zum Übergang vom Bachelor- ins Masterstudium: Welche Motive sind für die Wahl eines Masterstudiums entscheidend? In: Beiträge zur Hochschulforschung 33, 3, 40-61

Heine, C. (2012): Übergang vom Bachelor- zum Masterstudium. Hannover. = Studien zum deutschen Innovationssystem 2-2012.

Hildenbrand, A.; Soviana, S. (2015): Über die Kriterien bei und die Zufriedenheit mit der Wahl eines Master-Studiengangs am Beispiel der agrarwissenschaftlichen Master-Studiengänge der Justus-Liebig-Universität Gießen. In: German Journal of Agricultural Economics $64,1,42-57$.

HRK - Hochschulrektorenkonferenz (2008): Mobilität im Studium. Eine Untersuchung zu Mobilität und Mobilitätshindernissen in gestuften Studiengängen innerhalb Deutschlands. Ergebnisse einer repräsentativen Befragung von HIS - Hochschul-Informations-System GmbH im Auftrag und in Zusammenarbeit mit der HRK. Bonn. = Statistik zur Hochschulpolitik 2/2008.

HRK - Hochschulrektorenkonferenz (2015): Statistische Daten zu Studienangeboten an Hochschulen in Deutschland. Studiengänge, Studierende, Absolventinnen und Absolventen. Bonn.

Jaeger, A.; Kopper, J. (2014): Third mission potential in higher education: measuring the regional focus of different types of HEIs. In: Review of Regional Research 34, 2, 95-118. doi: 10.1007/s10037014-0091-3

Konegen-Grenier, C. (2012): Die Bologna-Reform. Eine Zwischenbilanz zur Neuordnung der Studiengänge in Deutschland. Köln. = IW-Positionen - Beiträge zur Ordnungspolitik 53 .

Krabel, S.; Flöther, C. (2014): Here Today, Gone Tomorrow? Regional Labour Mobility of German University Graduates. In: Regional Studies 48, 10, 1609-1627. doi: 10.1080/00343404.2012.739282

Kratz, F.; Lenz, T. (2015): Regional-ökonomische Effekte von Hochschulabsolventen. In: Beiträge zur Hochschulforschung 37, 2, 8-27.

Krawietz, M. (2008): Studentische Mobilität in Deutschland - Der Einfluss der neuen Studiengänge Bachelor und Master. In: Bildung und Erziehung 61, 4, 387-411. doi: 10.7788/bue.2008.61.4.387

Kujath, H. J.; Stein, A. (2009): Rekonfigurierung des Raumes in der Wissensgesellschaft. In: Raumforschung und Raumordnung 67 5-6, 369-382. doi: 10.1007/BF03185712

Lörz, M. (2008): Räumliche Mobilität beim Übergang ins Studium und im Studienverlauf: Herkunftsspezifische Unterschiede in der Wahl und Nachhaltigkeit des Studienortes. In: Bildung und Erziehung 61, 4, 413-436.

McClelland, R. J.; Gandy, R. J. (2012): Undergraduate regional migration in the UK. Perspectives on local markets and trends for gender and international student groups. In: Studies in Higher Education 37, 8, 901-924. doi: 10.1080/03075079.2011.602397

Montgomery, M. (2002): A nested logit model of the choice of a graduate business school. In: Economics of Education Review 21, 5, 471-480.

Pietzonka, M. (2014): Gestaltung von Studiengängen im Zeichen von Bologna. Die Umsetzung der Studienreform und die Wirksamkeit der Akkreditierung. Wiesbaden. doi: 10.1007/978-3-658-06488-4

Prenzel, M. (2015): Der regionale Verbund als hochschulpolitische Perspektive. Dresden. https://www.wissenschaftsrat.de/ download/archiv/VS_Bericht_Juli_2014.pdf (29.09.2017).

Sage, J.; Evandrou; M.; Falkingham, J. (2013): Onwards or Homewards? Complex Graduate Migration Pathways, Well-being, and the 'Parental Safety Net'. In: Population, Space and Place 19, 6, 738-755. doi: 10.1002/psp.1793

Schubert, T.; Kroll, H. (2014): Universities' effects on regional GDP and unemployment: The case of Germany. In: Papers in Regional Science 95, 3, 467-489. doi: 10.1111/pirs. 12150

Singleton, A. D.; Wilson, A. G.; O'Brien, O. (2012): Geodemographics and spatial interaction: an integrated model for higher education. In: Journal of Geographic Systems 14, 2, 223-241. doi: 10.1007/s10109-010-0141-5

Studierendensekretariat Gießen (2017): Anonymisierte Studierendenstatistik der Justus-Liebig-Universität Gießen. Gießen.

Tarazona, M. (2010): Regionale Bildungsdisparitäten und Beschäftigungsentwicklung. In: Raumforschung und Raumordnung 68, 6, 471-481. 